\title{
The CHADS2 Score Predicts Ischemic Stroke in the Absence of Atrial Fibrillation Among Patients with Coronary Heart Disease: Data from the Heart and Soul Study
}

\author{
Christine C. Welles, MD ${ }^{1}$, Mary A. Whooley, MD ${ }^{1,2,3}$, Beeya Na, MPH ${ }^{2}$, Peter Ganz, MD ${ }^{1,4}$, \\ Nelson B. Schiller, MD ${ }^{1}$, and Mintu P. Turakhia, MD, MAS 5,6 \\ ${ }^{1}$ Department of Medicine, University of California, San Francisco, CA, USA \\ ${ }^{2}$ Veterans Affairs Medical Center, San Francisco, CA, USA \\ ${ }^{3}$ Department of Epidemiology and Biostatistics, University of California, San Francisco CA, USA \\ ${ }^{4}$ Division of Cardiology, San Francisco General Hospital, San Francisco, CA, USA \\ ${ }^{5}$ Center for Health Care Evaluation, Veterans Affairs Palo Alto Health Care System, Palo Alto, \\ CA, USA \\ ${ }^{6}$ Department of Medicine (Cardiovascular Medicine), Stanford University, Stanford, CA, USA
}

\begin{abstract}
Background-We sought to evaluate the prognostic performance of the CHADS2 score for prediction of ischemic stroke/TIA in subjects with coronary heart disease (CHD) without atrial fibrillation $(\mathrm{AF})$.
\end{abstract}

\begin{abstract}
Methods-In 916 non-anticoagulated outpatients with stable CHD and no AF by baseline electrocardiogram, we calculated CHADS2 scores (congestive heart failure, hypertension, age $\geq$ 75 , diabetes (1 point each), and prior stroke or transient ischemic attack (TIA) (2 points)). The primary outcome was time to ischemic stroke or TIA over a mean follow-up of $6.4 \pm 2.3$ years.
\end{abstract}

Results-Over 5821 person-years of follow-up, 40 subjects suffered an ischemic stroke/TIA (rate 0.69 per 100 person-years, 95\% CI 0.50-0.94). Compared to subjects with low (0-1) CHADS2 scores, those with intermediate (2-3) and high (4-6) CHADS2 scores had an increased rate of stroke/TIA, even after adjustment for age, tobacco, antiplatelet therapy, statins, and angiotensin inhibitors (CHADS2 score 2-3: HR 2.4, 95\% CI 1.1-5.3, p=0.03, CHADS2 score 4-6: HR 4.0, 95\% CI 1.5-10.6, $\mathrm{p}=0.006$ ). Model discrimination $(\mathrm{c}$-statistic $=0.65)$ was comparable to CHADS2 model fit in published AF-only cohorts.

Conclusions-The CHADS2 score predicts ischemic stroke/TIA in subjects with stable CHD and no baseline AF. The event rate in non-AF subjects with high CHADS2 scores (5-6) was comparable to published rates in AF patients with moderate CHADS2 scores (1-2), a population known to derive benefit from stroke prevention therapies. These findings should inform efforts to determine whether stroke prevention therapies or screening for silent AF may benefit subjects with stable CHD and high CHADS2 scores.

\section{Keywords}

CHADS2; Stroke; Atrial Fibrillation; Coronary Heart Disease; Risk Stratification

Address for Correspondence: Mintu Turakhia, M.D. M.A.S Palo Alto VA Health Care System Stanford University 3801 Miranda Ave - 111C Palo Alto CA 94304 Tel (650) 858-3932 Fax (866) 756-3025 mintu@ stanford.edu.

Disclosures: None 


\section{Introduction}

The CHADS2 score is a validated clinical prediction tool commonly used to estimate the risk of stroke in atrial fibrillation (AF). The score is derived from the sum of point values of individual stroke risk factors (congestive heart failure (CHF), hypertension, age $\geq 75$, diabetes (1 point each), and prior stroke or transient ischemic attack (TIA) (2 points)). ${ }^{1}$ The CHADS2 score is used in clinical practice to guide decisions regarding antiplatelet and anticoagulation therapy. The simplicity of its calculation has facilitated its widespread adoption and endorsement by national and international society guidelines. ${ }^{2-4}$

Although the CHADS2 score and other similar risk stratification schemes have proven useful in populations with known AF, the vast majority (85\%) of ischemic strokes occur in individuals without known AF. ${ }^{5}$ Patients with coronary heart disease (CHD) are at increased risk for stroke, and each of the component comorbidities of the CHADS2 score has been independently associated with stroke in large cohorts of patients with CHD. ${ }^{6-8}$ Therefore, we hypothesized that stroke risk may also be well captured by the CHADS2 score in the non-AF CHD population. To test this hypothesis, we evaluated the prognostic performance of the CHADS2 score for prediction of ischemic stroke/TIA in patients with CHD without AF.

\section{Methods}

\section{Participants}

The Heart and Soul Study is a prospective cohort study designed to investigate psychosocial factors and health outcomes in patients with stable CHD. Details regarding recruitment methods and study design have been published previously ${ }^{9}$. In brief, between September 2000 to December 2002, 1,024 outpatients with stable CHD were recruited from two Veterans Administration Medical Centers (Palo Alto and San Francisco), one university medical center (University of California, San Francisco), and nine clinics in the Community Health Network of San Francisco. Inclusion criteria were defined as meeting one or more of the following: (1) history of myocardial infarction (MI); (2) evidence of at least 50\% stenosis in 1 or more coronary vessels on cardiac catheterization; (3) evidence of exerciseinduced ischemia by treadmill electrocardiogram (EKG) or nuclear perfusion stress imaging; or (4) a history of coronary revascularization. Exclusion criteria were defined as one or more of the following: history of MI in the previous 6 months, inability to walk 1 block, or planning to move out of the local area within 3 years.

Of 1,024 study subjects, we excluded participants with atrial fibrillation or flutter on baseline EKG ( $n=50)$, warfarin use ( $n=46)$, missing data for calculation of the CHADS2 score $(n=8)$, and those lost to follow-up $(n=4)$. The remaining 916 participants are the subjects of this secondary data analysis. This study was approved by the institutional review board and all participants provided written, informed consent.

\section{Measurements}

Predictor Variable-The primary predictor was the CHADS2 score. Hypertension was defined as either self-report or systolic blood pressure $\geq 160 \mathrm{mmHg}$; blood pressure was measured in all participants in the supine position after five minutes of rest. This cutpoint was chosen because it was the criterion used by the Atrial Fibrillation Investigator study $(\mathrm{AFI})^{10}$, one of the two classification schemes amalgamated to form the CHADS2 index. Diabetes was defined as self-reported diabetes, receipt of a diabetes medication, or hemoglobin A1c $\geq 7.0 \%$. CHF, prior stroke and TIA were determined by self-report. 
Outcome Variable-The primary outcome was time to incident TIA or ischemic stroke. Annual follow-up interviews with participants or their proxy were conducted to inquire about interval death or hospitalization. For any reported event, medical records were retrieved and reviewed by two independent and blinded physician adjudicators. If the adjudicators agreed on the outcome classification, their classification was binding. In the event of a disagreement, a third blinded adjudicator was consulted.

Stroke was defined as a new neurologic deficit not known to be secondary to brain trauma, tumor, infection, or other cause, based on the WHO MONICA criteria. ${ }^{11,12}$ All stroke outcomes were subtyped as hemorrhagic, ischemic, or procedure-related, based on physician diagnosis, which was confirmed by CT/MRI imaging in $84 \%$ of cases. Stroke outcomes in this study were restricted to patients with non-procedure-related ischemic strokes. TIA outcomes were based on the clinical judgment of the adjudicators who independently reviewed the medical records (including medical history, physical examination, imaging, laboratory results, and diagnosis of the treating physician), guided by the definition of TIA as a focal neurologic deficit (in the absence of head trauma) lasting more than 30 seconds and no longer than 24 hours, with rapid evolution of the symptoms to the maximal level of deficit in less than 5 minutes and with subsequent complete resolution.

Other Measurements-Age, sex, race, and medical history were determined by selfreported questionnaire. Height and weight were measured to calculate body mass index (BMI). Participants were instructed to bring all medication bottles to the baseline appointment, and study personnel recorded all current medications. Standard 12-lead EKGs were performed on all subjects at the time of enrollment. EKGs were adjudicated by two independent, blinded physicians. In the event of a disagreement, a third adjudicator was consulted. In addition, all EKGs with a paced rhythm were reviewed by a cardiac electrophysiologist to rule out underlying AF.

\section{Statistical Analysis}

For descriptive purposes, we divided the primary predictor, the CHADS2 score, into 3 strata of risk: low (0-1), intermediate (2-3) and high (4-6). We compared differences in baseline characteristics between patients with low, intermediate, and high CHADS2 scores using chisquare tests for categorical variables and one-way analysis of variance (ANOVA) for continuous variables.

We calculated cumulative event incidence rates by CHADS2 score. We then used Cox regression to calculate the risk of ischemic stroke or TIA, stratified by CHADS2 score, for comparison with the hazard ratios reported in the original CHADS2 derivation study. ${ }^{1} \mathrm{We}$ measured cumulative event-free survival by the Kaplan-Meier method and compared unadjusted differences using the log-rank test. We performed Cox regression to assess the ability of the CHADS2 score to independently predict incident stroke/TIA after multivariate adjustment. To avoid model overfitting with the relatively small number of outcome events, we limited adjustment to five covariates based on prior literature and clinical validity: age ${ }^{5}$ and current smoking ${ }^{13}$ (known stroke risk factors), statins ${ }^{14}$ and antiplatelet agents ${ }^{15}$ (proven stroke prevention therapies), and angiotensin inhibitors ${ }^{16,17}$ (may prevent left atrial remodeling/AF and improve ventricular function). To assess the potential for residual confounding, we also performed a sensitivity analysis in which all covariates demonstrating an association with the CHADS2 score at $\mathrm{p}<0.05$ were included in the model. The assumption of proportional hazards was found to be valid using log-minus-log curves and the Schoenfeld test ${ }^{18}, 19$. Finally, to compare discrimination of the CHADS2 score with other similar clinical stroke risk stratification models, we calculated c-statistics for the 
$\mathrm{CHA}_{2} \mathrm{DS}_{2}-\mathrm{VASc}^{20}$ and Framingham stroke risk scores. ${ }^{21,}{ }^{22}$ All analyses were conducted using STATA, version 11.0 (College Station, TX).

Dr. Welles was supported by a National Research Service Award (1-T32-HP-19025). Dr. Turakhia was supported by a Veterans Health Services Research \& Development Career Development Award (CDA09027-1) and an American Heart Association National Scientist Development Grant (09SDG2250647). The Heart and Soul Study was supported by the Department of Veterans Affairs, the National Heart, Lung, and Blood Institute, the American Federation for Aging Research, the Robert Wood Johnson Foundation, and the Ischemia Research and Education Foundation. The content and opinions expressed are solely the responsibility of the authors and do not necessarily represent the views or policies of the Department of Veterans Affairs. The authors are solely responsible for the design and conduct of this study, all study analyses and drafting and editing of the paper.

\section{Results}

Of the 916 non-anticoagulated participants with no evidence of baseline AF, the mean CHADS2 score was $1.7 \pm 1.2$. The CHADS2 score was strongly associated with each of the components used to derive the score (age, CHF, hypertension, diabetes, stroke/TIA). Compared with participants with low CHADS2 scores (0-1), those with high CHADS2 scores (4-6) also had higher BMIs and were less likely to smoke. They were more likely to have a history of MI, lower left ventricular ejection fractions (LVEF), and higher values of systolic blood pressure, hemoglobin A1c and serum creatinine. Finally, participants with higher CHADS2 scores were more likely to be using beta blocker, statin, angiotensin inhibitor, antiplatelet and antiarrhythmic medications (Table I).

The primary outcome of ischemic stroke/TIA occurred in 40 participants during a mean follow-up time of $6.4 \pm 2.3$ years or 5821 person-years (rate 0.69 per 100 person-years, $95 \%$ CI 0.50-0.94). Event rates increased by CHADS2 score (0: 0.2, 1: 0.4, 2: 0.9, 3: 0.9, 4: 1.1, 5-6: 3.1 per 100 person-years, p for trend $=0.0002)($ Table II, Figure 1).

Kaplan-Meier estimates revealed early separation of the event-free survival curves (within the first six months of follow-up), which continued to diverge throughout follow-up (Figure 2). Compared with the reference group of subjects with CHADS2 scores of 0-1, those with CHADS2 scores of 2-3 had 2.7 times the rate of stroke/TIA ( $\mathrm{p}=0.01$ ), and those with CHADS2 scores of 4-6 had 4.6 times the rate ( $\mathrm{p}=0.001$ ). After adjustment for age, smoking, use of statins, antiplatelet agents and angiotensin inhibitors, CHADS2 score remained independently associated with time to stroke/TIA (CHADS2 score 2-3: HR 2.4, p=0.03, CHADS2 score 4-6: HR 4.0, $\mathrm{p}=0.006$ ). We also performed a sensitivity analysis, in which we created an expanded multivariate model where we also adjusted for serum creatinine, BMI, prior MI, and use of antiarrhythmic agents. After adjustment for these additional covariates, the associations remained significant and similar in magnitude (Table III).

We performed a subgroup analysis limited to only patients with no prior stroke/TIA. In our subgroup analysis, the point estimates for the hazard ratios of patients with CHADS2 scores 2-3 were were similar to those of the main analysis (subgroup analysis: 2.6 (1.1-5.8), $\mathrm{p}=0.03$, compared with main analysis: $2.7(1.3-5.7), \mathrm{p}=0.01)$, and there was no interaction ( $\mathrm{p}$ $=0.73$ ).

In our cohort, test discrimination of the CHADS2 score (c-statistic $=0.65)$ was similar to discrimination when applying other clinical stroke risk stratification models: $\mathrm{CHA}_{2} \mathrm{DS}_{2^{-}}$ $\operatorname{VASc}^{20}(0.64)$, Framingham stroke risk score for patients with no AF (0.63) and new-onset AF (0.64). Because patients with higher CHADS2 scores had lower LVEFs, we also created 
a separate model in which CHF was defined by either self-report or LVEF $\leq 40 \%$ and found no appreciable change in the c-statistic (0.65).

\section{Discussion}

In 916 patients with coronary heart disease who had no known $\mathrm{AF}$ at baseline, we found that the CHADS2 score was strongly predictive of ischemic stroke/TIA. Compared to participants with low CHADS2 scores (0-1), those with intermediate CHADS2 scores (2-3) had an over 2-fold increased rate of stroke and those with high CHADS2 scores (4-6) had a 4-fold increased rate of stroke. The event rate in non-AF CHD patients with high CHADS2 scores (5-6) was comparable to the rate in AF patients with moderate CHADS2 scores (1-2), a population known to derive benefit from stroke prevention therapies such as anticoagulation. ${ }^{15}$ This association remained independent after adjustment for a wide range of covariates. Moreover, model discrimination in this non-AF cohort (c-statistic $=0.65)$ was similar to that which has been observed in external contemporary AF cohorts (c-statistic $=$ 0.56-0.62). ${ }^{23}$ These findings indicate the CHADS2 score may also be useful for risk stratification of patients without known AF. Notably, the discrimination of other, more complex risk stratification schemes $\left(\mathrm{CHA}_{2} \mathrm{DS}_{2}-\mathrm{VASc}\right.$, Framingham) were no better than CHADS2 in this population $(\mathrm{c}$-statistic $=0.63-0.64)$.

Several potential mechanisms may explain these findings. First, patients with high CHADS2 scores may have a higher risk of developing atrial arrhythmias, which mediates the relationship with stroke outcomes. The strongest support for this comes from the cryptogenic stroke literature. In a study of patients monitored for arrhythmia after suffering an ischemic stroke, higher CHADS2 scores were noted in patients subsequently found to have occult AF. This association was particularly strong in patients with CHD. These investigators suggested using the CHADS2 criteria "backwards" to identify a subset of patients with cryptogenic stroke who may benefit from prolonged event monitoring. ${ }^{24} \mathrm{~A}$ similar investigation of hypertensive patients admitted for ischemic stroke demonstrated a higher prevalence of $\mathrm{CHF}$, diabetes, $\mathrm{CHD}$, and advanced age in patients found to have silent $\mathrm{AF}$ than those in whom monitoring did not reveal $\mathrm{AF}^{25}$

Alternatively, the CHADS2 risk factors themselves may increase the risk of stroke or stroke subtypes, independent of cardiac rhythm. In AF, substantial evidence supports induction of a prothrombotic state, endothelial dysfunction, and blood stasis as underlying mechanisms of thrombus formation and stroke. ${ }^{26}$ However, even in the absence of AF, patients with heart failure ${ }^{27}$, hypertension, ${ }^{28}$ and diabetes ${ }^{29}$ have elevated markers of hypercoagulability and endothelial dysfunction. A recent study demonstrated similar levels of platelet activation in both $\mathrm{AF}$ and non-AF patients with cardiovascular comorbidities, suggesting that platelet activation in AF may be due to underlying cardiovascular disease rather than AF itself. ${ }^{30}$

Finally, the CHADS2 risk factors may directly contribute to left atrial (LA) remodeling, a process characterized by dilatation and mechanical dysfunction of the left atrium. ${ }^{31}$ These factors may result in blood stasis and confer an increased risk of thromboembolism independent of rhythm. ${ }^{32}$ Atrial structural remodeling can be accompanied by wall stretch and electrical remodeling resulting in atrial arrhythmias, with AF being the most common. ${ }^{31,33,34}$ Diabetes, heart failure, hypertension, and CHD have all been associated with LA remodeling. ${ }^{35-37}$ The CHADS2 risk factors may therefore contribute to stroke risk via LA remodeling, either by chamber dilatation producing blood stasis, by induction of $\mathrm{AF}$, or both.

Further investigation is warranted to gain a better understanding of the underlying mechanism, as such knowledge may inform more directed screening or stroke prevention 
efforts. Our findings of comparable stroke risk in non-AF patients with high CHADS2 scores (5-6) and AF patients with moderate CHADS2 scores (1-2) raise the question of whether high risk, non-AF patients may benefit from stroke prevention therapies such as anticoagulation, either due to a greater risk of silent AF or rhythm-independent mechanisms of thromboembolism in this population.

The 2011 AHA/ASA guidelines for prevention of primary and recurrent stroke recommend risk factor modification of hyperlipidemia, hypertension, and diabetes. ${ }^{38,39}$ For patients with CHD or prior stroke/TIA, the guidelines recommend antiplatelet therapy. Notably, there were stroke events in our cohort despite the fact that the majority of subjects were receiving antiplatelet therapies (84\%) and statins $(65 \%)$ and had achieved excellent risk factor control (low density lipoprotein: $105+/-33 \mathrm{mg} / \mathrm{dL}$, systolic blood pressure: $133+/-21 \mathrm{mmHg}$, hemoglobin A1c: $6.0+/-1.2 \%$ ). Although stroke prevention guidelines state that opportunistic pulse screening followed by EKG to assess for AF in patients $\geq 65$ may be useful, this limited ascertainment may be insensitive for sufficient detection of paroxysmal AF. 39,40

There are several notable limitations of our study. This study was performed in a cohort of predominantly male patients with stable CHD, which may limit generalizability. The observational nature of the study cannot eliminate the possibility of residual confounding. However, the CHADS2 score remained strongly and independently predictive of stroke/TIA after adjustment for potential confounding variables; more extensive adjustment did not change point estimates or widen confidence intervals. Notably, approximately $6.2 \%$ of participants in the study were receiving antiarrhythmic agents, with greater use in those with high CHADS2 scores. If prescribed for atrial rather than ventricular arrhythmias, those with high CHADS2 scores may have had a higher rate of paroxysmal AF at baseline. Adjustment for antiarrhythmic drug use demonstrated no change in point estimates.

Finally, because our criterion for exclusion of subjects with AF was the baseline EKG, it is possible that individuals with known paroxysmal AF who were in sinus rhythm at the time of the baseline EKG could have been misclassified as non-AF participants and included in the analyses. It is also possible that participants without known AF but who had AF on the baseline study EKG could have been misclassified and excluded. However, for paroxysmal $\mathrm{AF}$, any method of AF ascertainment other than continuous EKG monitoring would be subject to similar misclassification.

\section{Conclusions}

In summary, we found that the CHADS2 risk score independently predicts stroke/TIA in patients with CHD who have no known history of AF. The event rate in non-AF CHD patients with high CHADS2 scores (5-6) was comparable to published rates in AF patients with moderate CHADS2 scores (1-2). Since most strokes occur in patients without known $\mathrm{AF}$, the CHADS2 score may have a role in identification of high-risk individuals who may benefit from stroke prevention therapies or screening for silent AF.

\section{References}

1. Gage BF. Validation of clinical classification schemes for predicting stroke: Results from the National Registry of Atrial Fibrillation. Jama. 2001; 285:2864-2870. [PubMed: 11401607]

2. Camm AJ, Kirchhof P, Lip GYH, et al. Guidelines for the management of atrial fibrillation. European Heart Journal. 2010; 31:2369-2429. [PubMed: 20802247]

3. Singer D, Albers G, Dalen J, et al. American College of Chest Physicians. Antithrombotic therapy in atrial fibrillation: American College of Chest Physicians evidence-based clinical practice guidelines. Chest. 2008; 133:546S-592S. 
4. Fuster V, Ryden L, Cannom D, et al. ACC/AHA/ESC 2006 guidelines for the management of patients with atrial fibrillation: A report of the American College of Cardiology/American Heart Association task force on practice guidelines and the European Society of Cardiology committee for practice guidelines (writing committee to revise the 2001 guidelines for the management of patients with atrial fibrillation): Developed in collaboration with the European Heart Rhythm association and the Heart Rhythm Society. Circulation. 2006; 114:e257. [PubMed: 16908781]

5. Lloyd-Jones D, Adams R, Carnethon M, et al. Heart disease and stroke statistics--2009 update: A report from the American Heart Association statistics committee and stroke statistics subcommittee. Circulation. 2009; 119:e21-181. [PubMed: 19075105]

6. Yusuf S, Sleight P, Pogue J, et al. Heart Outcomes Prevention Evaluation Study. Effects of an angiotensin-converting-enzyme inhibitor, ramipril, on cardiovascular events in high-risk patients. N Engl J Med. 2000; 342:145-153. [PubMed: 10639539]

7. Kannel WB, McGee DL. Diabetes and cardiovascular disease - Framingham Study. Jama. 1979; 241:2035-2038. [PubMed: 430798]

8. Loh E, Sutton MSJ, Wun CCC, et al. Ventricular dysfunction and the risk of stroke after myocardial infarction. N Engl J Med. 1997; 336:251-257. [PubMed: 8995087]

9. Ruo B, Rumsfeld JS, Hlatky MA, et al. Depressive symptoms and health-related quality of life: The Heart and Soul Study. Jama. 2003; 290:215-221. [PubMed: 12851276]

10. Atrial Fibrillation Investigators: Atrial Fibrillation A, Anticoagulation Study, Boston Area Anticoagulation Trial for Atrial Fibrillation Study, Canadian Atrial Fibrillation Anticoagulation Study, Stroke Prevention in Atrial Fibrillation Study, Veterans Affairs Stroke Prevention in Nonrheumatic Atrial Fibrillation Study. Risk factors for stroke and efficacy of antithrombotic therapy in atrial fibrillation: Analysis of pooled data from five randomized controlled trials. Arch Intern Med. 1994; 154:1449-1457. [PubMed: 8018000]

11. Asplund K, Bonita R, Kuulasmaa K, et al. Multinational comparisons of stroke epidemiology: Evaluation of case ascertainment in the WHO MONICA stroke study. Stroke. 1995; 26:355. [PubMed: 7886706]

12. Thorvaldsen P, Asplund K, Kuulasmaa K, et al. Stroke incidence, case fatality, and mortality in the WHO MONICA project. Stroke. 1995; 26:361. [PubMed: 7886707]

13. Wolf P, D'Agostino R, Kannel W, et al. Cigarette smoking as a risk factor for stroke: The Framingham Study. Jama. 1988; 259:1025. [PubMed: 3339799]

14. Bucher HC, Griffith LE, Guyatt GH. Effect of HMG-CoA reductase inhibitors on stroke. Ann Intern Med. 1998; 128:89-95. [PubMed: 9441587]

15. Hart RG, Benavente O, McBride R, et al. Antithrombotic therapy to prevent stroke in patients with atrial fibrillation: A meta-analysis. Ann Intern Med. 1999; 131:492. [PubMed: 10507957]

16. Nakashima H, Kumagai K, Urata H, et al. Angiotensin II antagonist prevents electrical remodeling in atrial fibrillation. Circulation. 2000; 101:2612. [PubMed: 10840013]

17. Tsang T, Barnes M, Abhayaratna W, et al. Effects of quinapril on left atrial structural remodeling and arterial stiffness. Am J Cardiol. 2006; 97:916-920. [PubMed: 16516602]

18. Schoenfeld D. Partial residuals for the proportional hazards regression model. Biometrika. 1982; 69:239-241.

19. Grambsch P, Therneau T. Proportional hazards tests and diagnostics based on weighted residuals. Biometrika. 1994; 81:515.

20. Lip G, Nieuwlaat R, Pisters R, et al. Refining clinical risk stratification for predicting stroke and thromboembolism in atrial fibrillation using a novel risk factor-based approach. Chest. 2010; 137:263. [PubMed: 19762550]

21. D’Agostino R, Wolf P, Belanger A, et al. Stroke risk profile: Adjustment for antihypertensive medication. The Framingham Study. Stroke. 1994; 25:40. [PubMed: 8266381]

22. Wang T, Massaro J, Levy D, et al. A risk score for predicting stroke or death in individuals with new-onset atrial fibrillation in the community: The Framingham Heart Study. Jama. 2003; 290:1049. [PubMed: 12941677]

23. Fang M, Go A, Chang Y, et al. Comparison of risk stratification schemes to predict thromboembolism in people with nonvalvular atrial fibrillation. J Am Coll Cardiol. 2008; 51:810815. [PubMed: 18294564] 
24. Haft JI. Using chads2 backwards plus echo criteria to identify stroke patients who have occult intermittent atrial fibrillation. Am Heart J. 2009; 157:e9-e9. [PubMed: 19185625]

25. Haft JI, Teichhoz LE. Echocardiographic and clinical risk factors for atrial fibrillation in hypertensive patients with ischemic stroke. Am J Cardiol. 2008; 102:1348-1351. [PubMed: 18993153]

26. Watson T, Shantsila E, Lip G. Mechanisms of thrombogenesis in atrial fibrillation: Virchow's triad revisited. Lancet. 2009; 373:155. [PubMed: 19135613]

27. Chong A, Blann A, Patel J, et al. Endothelial dysfunction and damage in congestive heart failure: Relation of flow-mediated dilation to circulating endothelial cells, plasma indexes of endothelial damage, and brain natriuretic peptide. Circulation. 2004; 110:1794. [PubMed: 15364797]

28. Gregory YHL, Andrew DB, Alan FJ, et al. Relation of endothelium, thrombogenesis, and hemorheology in systemic hypertension to ethnicity and left ventricular hypertrophy. Am J Cardiol. 1997; 80:1566-1571. [PubMed: 9416937]

29. McClung J, Naseer N, Saleem M, et al. Circulating endothelial cells are elevated in patients with type 2 diabetes mellitus independently of HbA1c. Diabetologia. 2005; 48:345-350. [PubMed: 15660261]

30. Choudhury A, Chung I, Blann AD, et al. Platelet surface cd62p and cd63, mean platelet volume, and soluble/platelet $\mathrm{p}$-selectin as indexes of platelet function in atrial fibrillation - a comparison of "healthy control subjects" and "disease control subjects" in sinus rhythm. J Am Coll Cardiol. 2007; 49:1957-1964. [PubMed: 17498581]

31. Casaclang-Verzosa G, Gersh BJ, Tsang TSM. Structural and functional remodeling of the left atriumclinical and therapeutic implications for atrial fibrillation. J Am Coll Cardiol. 2008; 51:111. [PubMed: 18174029]

32. Barnes M, Miyasaka Y, Seward J, et al. Left atrial volume in the prediction of first ischemic stroke in an elderly cohort without atrial fibrillation. Mayo Clin Proc. 2004; 79:1008. [PubMed: 15301328]

33. Vaziri S, Larson M, Benjamin E, et al. Echocardiographic predictors of nonrheumatic atrial fibrillation. The Framingham Heart Study. Circulation. 1994; 89:724-730. [PubMed: 8313561]

34. Casaclang-Verzosa G, Barnes ME, Blume G, et al. C-reactive protein, left atrial volume, and atrial fibrillation: A prospective study in high-risk elderly. Echocardiography. 2010; 27:394-399. [PubMed: 20070356]

35. Peterson LR, Waggoner AD, de las Fuentes L, et al. Alterations in left ventricular structure and function in type-1 diabetics: A focus on left atrial contribution to function. J Am Soc of Echocardiogr. 2006; 19:749-755. [PubMed: 16762752]

36. Sanders P, Morton JB, Davidson NC, et al. Electrical remodeling of the atria in congestive heart failure: Electrophysiological and electroanatomic mapping in humans. Circulation. 2003; 108:1461-1468. [PubMed: 12952837]

37. Tsang TSM, Barnes ME, Gersh BJ, et al. Left atrial volume as a morphophysiologic expression of left ventricular diastolic dysfunction and relation to cardiovascular risk burden. Am J of Cardiol. 2002; 90:1284-1289. [PubMed: 12480035]

38. Furie K, Kasner S, Adams R, et al. Guidelines for the prevention of stroke in patients with stroke or transient ischemic attack. A guideline for healthcare professionals from the American Heart Association/American Stroke Association. Stroke. 2011; 42:227-276. [PubMed: 20966421]

39. Goldstein L, Bushnell C, Adams R, et al. Guidelines for the primary prevention of stroke. A guideline for healthcare professionals from the American Heart Association/American Stroke Association. Stroke. 2011; 42:227-276. [PubMed: 20966421]

40. Fitzmaurice DA, Hobbs F, Jowett $S$, et al. Screening versus routine practice in detection of atrial fibrillation in patients aged 65 or over: cluster randomised controlled trial. BMJ. 2007; 335:383386. [PubMed: 17673732] 


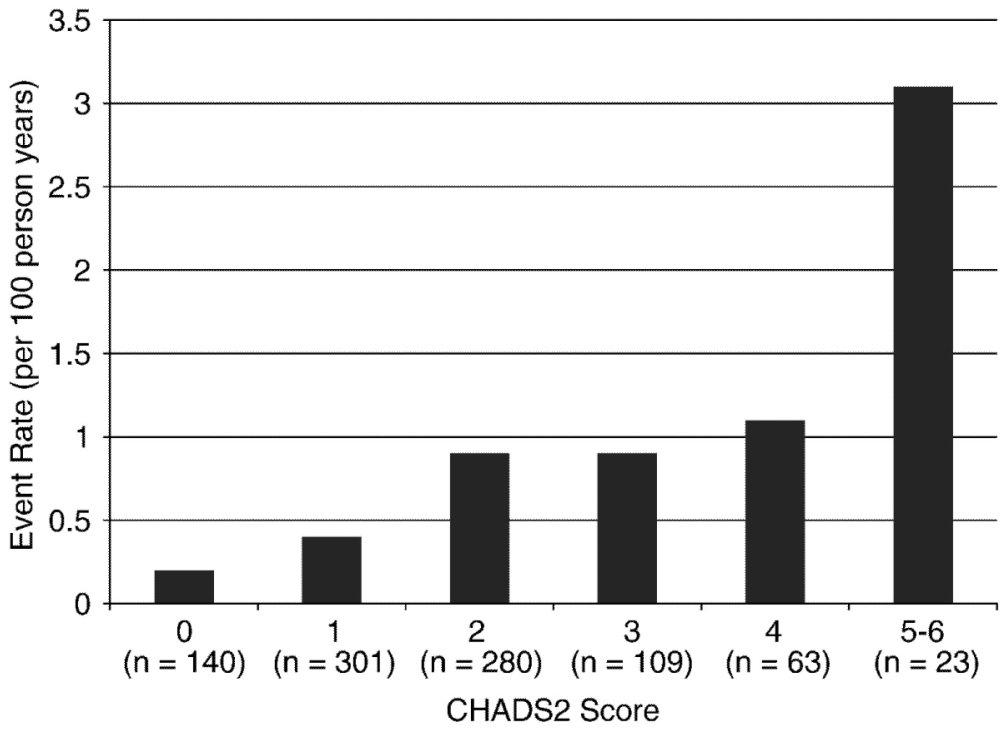

Figure 1.

Rate of ischemic stroke/TIA, by CHADS2 score 


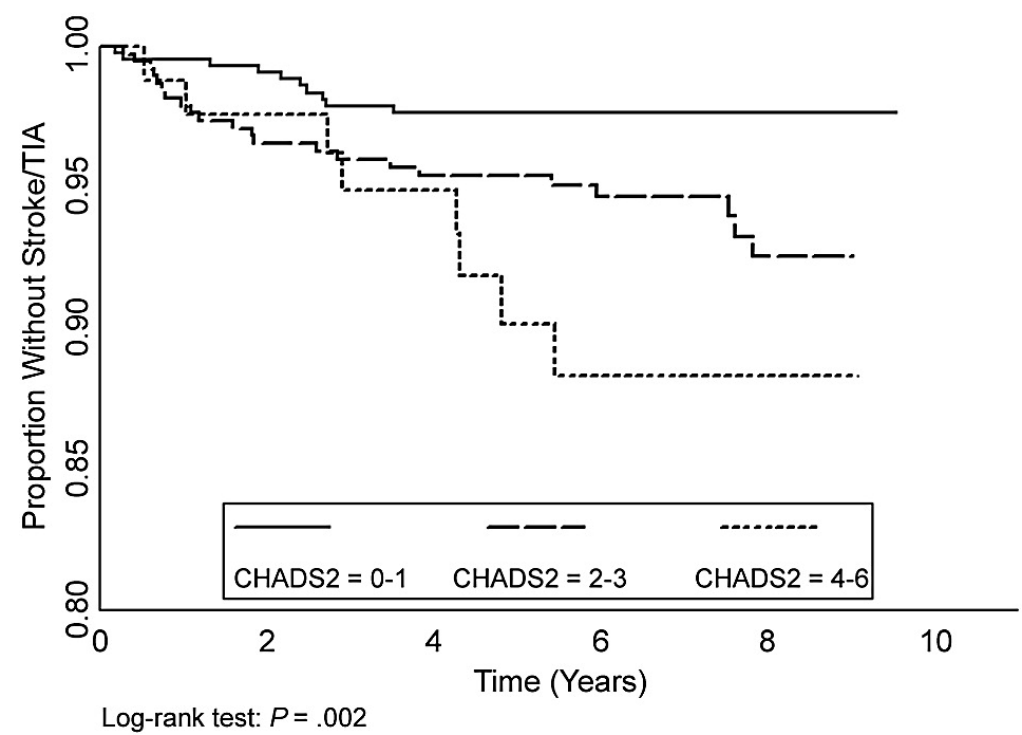

Figure 2.

Kaplan Meier curves for survival free of ischemic stroke/TIA, stratified by CHADS2 score 


\section{Table I}

Baseline characteristics of 916 participants with stable coronary heart disease and no baseline AF, by CHADS2 score

\begin{tabular}{|c|c|c|c|c|}
\hline & \multicolumn{3}{|c|}{ CHADS2 Score } & \multirow[b]{2}{*}{ p-value } \\
\hline & $\begin{array}{c}0-1 \\
(n=441)\end{array}$ & $\begin{array}{c}2-3 \\
(n=389)\end{array}$ & $\begin{array}{c}4-6 \\
(n=86)\end{array}$ & \\
\hline \multicolumn{5}{|l|}{ Demographics } \\
\hline Age, years & $63.5 \pm 9.6$ & $69.0 \pm 11.1$ & $72.9 \pm 9.7$ & 0.007 \\
\hline Male, $\%$ & 81.2 & 81.0 & 87.2 & 0.38 \\
\hline Caucasian, $\%$ & 62.5 & 56.0 & 52.3 & 0.07 \\
\hline Body Mass Index, kg/m² & $27.9 \pm 4.8$ & $28.9 \pm 5.6$ & $28.6 \pm 4.6$ & 0.01 \\
\hline Current Smoker, \% & 24.1 & 16.2 & 20.9 & 0.02 \\
\hline \multicolumn{5}{|l|}{ Medical History } \\
\hline Myocardial Infarction, \% & 48.1 & 56.9 & 69.8 & $<0.001$ \\
\hline Congestive Heart Failure, \% & 2.3 & 25.5 & 46.5 & $<0.001$ \\
\hline Hypertension, \% & 52.8 & 92.0 & 94.2 & $<0.001$ \\
\hline Diabetes, \% & 5.0 & 47.8 & 50.0 & $<0.001$ \\
\hline Prior Stroke/TIA, \% & 0.0 & 12.3 & 88.4 & $<0.001$ \\
\hline \multicolumn{5}{|l|}{ Laboratory/Imaging } \\
\hline $\mathrm{SBP}, \mathrm{mmHg}$ & $130 \pm 19$ & $137 \pm 22$ & $135 \pm 24$ & $<0.001$ \\
\hline Hemoglobin A1c, \% & 5.5 & 6.4 & 6.4 & $<0.001$ \\
\hline $\mathrm{HDL}, \mathrm{mg} / \mathrm{dL}$ & $47 \pm 15$ & $45 \pm 14$ & $45 \pm 13$ & 0.07 \\
\hline $\mathrm{LDL}, \mathrm{mg} / \mathrm{dL}$ & $106 \pm 33$ & $103 \pm 35$ & $104 \pm 33$ & 0.29 \\
\hline Serum Creatinine, $\mathrm{mg} / \mathrm{dL}$ & $1.0 \pm 0.4$ & $1.2 \pm 0.8$ & $1.3 \pm 0.6$ & $<0.001$ \\
\hline LVEF, \% & 62.8 & 61.0 & 61.4 & 0.03 \\
\hline \multicolumn{5}{|l|}{ Medication Use } \\
\hline Antiplatelet Agent ${ }^{*}, \%$ & 79.1 & 88.4 & 83.7 & 0.002 \\
\hline Beta Blocker, \% & 53.7 & 64.0 & 61.6 & 0.01 \\
\hline Angiotensin Inhibitor, \% & 38.1 & 62.7 & 57.0 & $<0.001$ \\
\hline Statin, \% & 59.6 & 67.6 & 75.6 & 0.004 \\
\hline Antiarrhythmics, \% & 3.6 & 8.2 & 10.5 & 0.005 \\
\hline
\end{tabular}

$\mathrm{TIA}=$ transient ischemic attack, $\mathrm{SBP}=$ systolic blood pressure, $\mathrm{HDL}=$ high density lipoprotein, $\mathrm{LDL}=$ low density lipoprotein, $\mathrm{LVEF}=$ left ventricular ejection fraction

Values are expressed as mean \pm standard deviation or percentage

* Aspirin \pm clopidigrel 


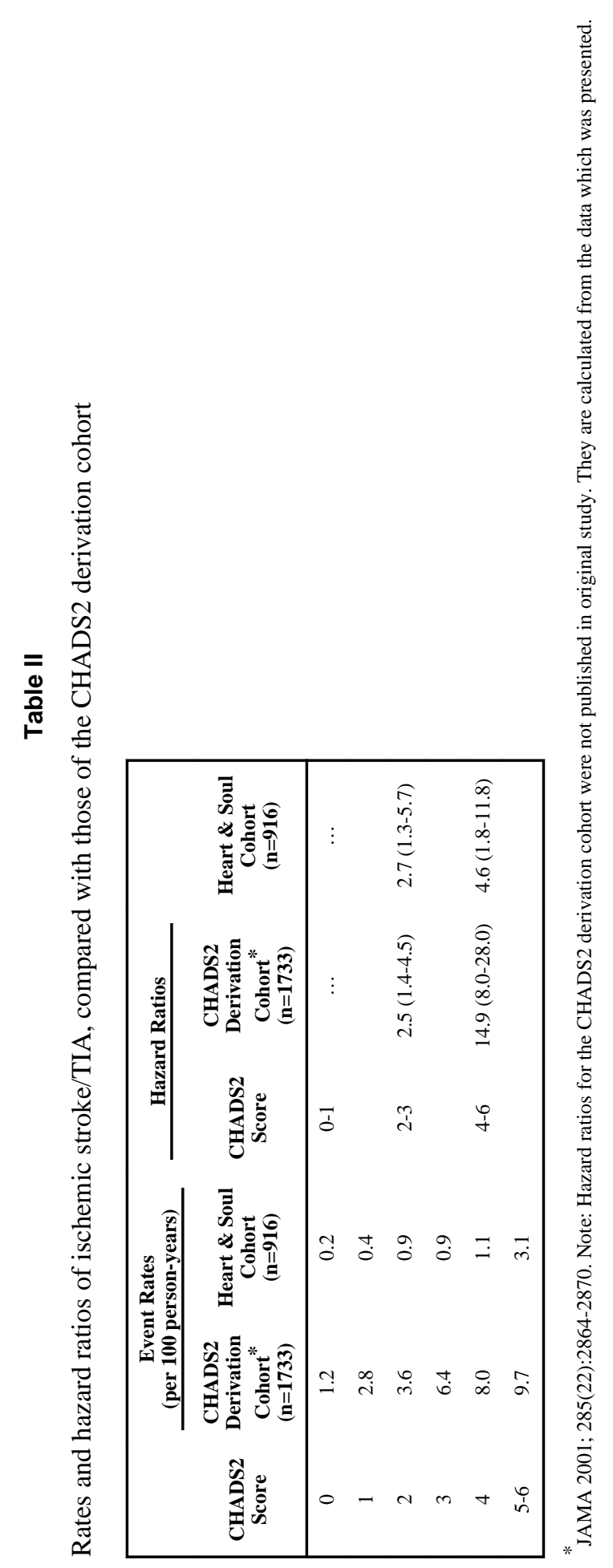

Am Heart J. Author manuscript; available in PMC 2012 September 1. 


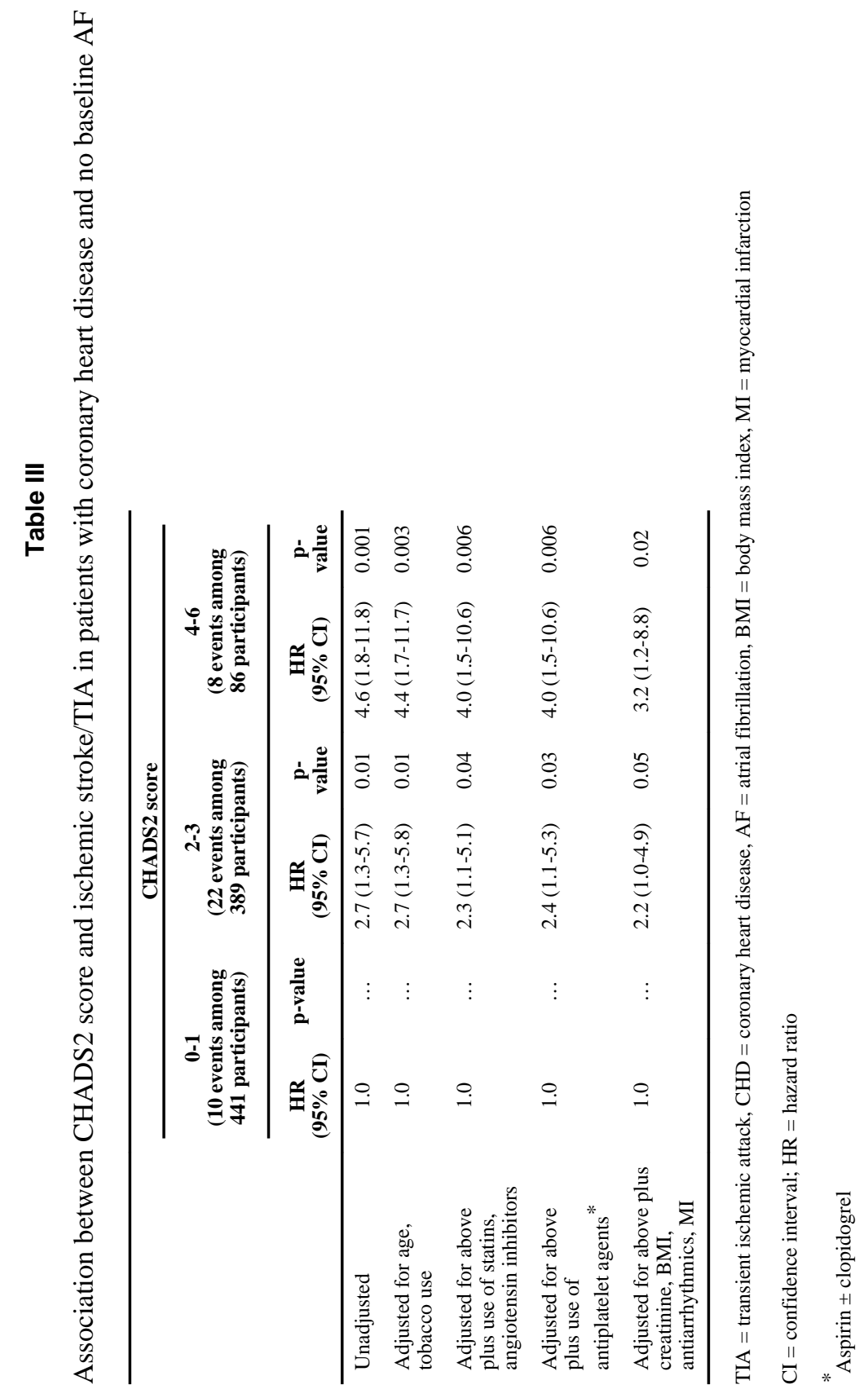

